\title{
Multiuser CDMA Parameters Estimation by Particle Filter with Resampling Schemes
}

\author{
Jang-Sub Kim ${ }^{1}$, Dong-Ryeol Shin ${ }^{1}$, and Woo-Gon Chung ${ }^{2}$ \\ ${ }^{1}$ School of Information and Communication Engineering, \\ Sungkyunkwan University, \\ 300 ChunChun-Dong, JangAn-Gu, Suwon, Korea 440-746 \\ \{jangsub,drshin\}@ece.skku.ac.kr \\ ${ }^{2}$ Computer Science Dept. CSU at Bakersfield, USA \\ wchung@csub.edu
}

\begin{abstract}
The joint estimation of linear and nonlinear state variables remains challenging, especially in multiuser communications applications where the state dimension is large and signal to noise ratio is low. In this paper, an efficient Particle Filter (PF) is developed to make estimates of nonlinear time delay parameters in the presence of non-Gaussian noise. The PF method has the advantage that the importance weights are easily evaluated and the importance density can be easily sampled. We propose a PF-based algorithm with Resampling schemes for the estimation of closely-spaced path delays and related coefficients in CDMA environments. Furthermore we present a number of resampling schemes, namely: Multinomial Resampling (MR), Residual Resampling (RR) and Minimum Variance Resamplings (MVR). The simulation results show that MR scheme outperforms the other selection schemes. We also show that it provides a more suitable method for tracking time-varying amplitudes and delays in CDMA communication systems than RR and MVR schemes.
\end{abstract}

\section{Introduction}

Nonlinearity in the measurement model has always been an obstacle for the reliability of the estimation in the Kalman filtering structure. Extended Kalman Filtering (EKF) approximates the a posteriori distribution to be Gaussian to simplify computation. Recently the unscented transform(UT) has been used in an EKF framework, and the resulting filter, known as the unscented Kalman filter, has been employed to tackle the nonlinearity and shown its effectiveness in terms of the divergence reduction or error propagation [1]. However, all the iterative solutions including Kalman filters appeared in the literature necessarily assume that the unknown parameters are Gaussian distributed.

In a CDMA environment which involves a joint channel coefficient and time-delay tracking techniques, non-Gaussian properties of unknown parameters are inherently taken. In this paper, the particle filter, recently introduced in the communication area, which does not require the Gaussian assumption is employed for such an exponentially distributed parameter like the propagation delay in the multipath environment [2]. The PF results in better performance in the parameter (including 
other channel coefficients) estimation [3]. We present PF with resampling techniques to get around degeneracy problem occurring in the PF without resampling schemes. Furthermore we demonstrate a number of resampling schemes, namely: Multinomial Resampling (MR), Residual Resampling (RR) and Minimum Variance Resamplings (MVR). The major contribution of this paper is to adapt the PF filter to the CDMA parameter estimation in the highly nonlinear environments to make easy and practical implementation by resampling techniques and to show performance comparisions among resampling techniques..

This paper is organized as follows. Section 2 introduces the signal and channel model that will be used throughout the paper and a description of problem formulation. Section 3 provides a description of PF and resampling schemes used for parameter estimation. The simulation and results of the performance from computer simulations are given in Section 4. Finally, Section 5 provides concluding remarks.

\section{Problem Formulation}

\subsection{System and Channel Model}

In DS spread-spectrum [2], the received baseband signal is given by

$$
r(l)=\sum_{k=1}^{K} \sum_{i=1}^{M} c_{k, i}(l) d_{k, m_{l}} a_{k}\left(l T_{s}-m_{l} T_{b}-\tau_{k, i}(l)\right)+n(l)
$$

where $\tau_{k, i}(l)$ and $c_{k, i}\left(l T_{s}\right)=\sqrt{P_{k}} f_{k, i}\left(l T_{s}\right) e^{j \phi_{k, i}\left(l T_{s}\right)}$ are delays and channel coefficient of $i$ th multipaths associated with the $k$ th user's channel, respectively. $a_{k}(t)$ and $d_{k, m}(l)$ are $k$ th user's PN spreading code and binary data sequence, respectively. AWGN, $n(l)$, is assumed to have zero mean and variance of $\sigma_{n}^{2}$.

\subsection{Problem Formulation}

Given the received waveform samples, $r(l)$, the task is to obtain minimum variance estimates of the unknown parameters $c_{k}$ and $\tau_{k}$ for $k=1,2, \cdots, K$, given by

$$
\hat{c}_{k}(l \mid l)=E\left\{c_{k}(l) \mid \mathbf{R}^{l}\right\} \quad \hat{\tau}_{k}(l \mid l)=E\left\{\tau_{k}(l) \mid \mathbf{R}^{l}\right\}
$$

where $\mathbf{R}^{l}\{r(l), r(l-1), \cdots, r(0)\}$ is the set of received samples up to time $l T_{s}$.

Let the unknown parameters be represented by the $2 K \times 1$ vector

$$
\mathbf{x}=\left[\begin{array}{ll}
\mathbf{c} & \boldsymbol{\tau}
\end{array}\right]^{T}
$$

where $\mathbf{c}=\left[c_{1}, c_{2}, \cdots, c_{K}\right]^{T}$ and $\boldsymbol{\tau}=\left[\tau_{1}, \tau_{2}, \cdots, \tau_{K}\right]^{T}$. 
By [2], we can write the state model as

$$
\mathbf{x}(l+1)=\mathbf{F}(l) \mathbf{x}(l)+\mathbf{v}(l)
$$

where the state $\mathbf{x}_{l}=\mathbf{x}(l)$ and $\mathbf{F}=\operatorname{diag}\left\{\mathbf{F}_{c}, \mathbf{F}_{\tau}\right\}$ is $2 K \times 2 K$ augmented state transition matrix, $\mathbf{v}=\left\lfloor\begin{array}{ll}\mathbf{v}_{c}^{T} & \mathbf{v}_{\tau}^{T}\end{array}\right\rfloor$ is $2 K \times 1$ process noise vector with zero mean and covariance matrix $\mathbf{Q}=\operatorname{diag}\left\{\mathbf{Q}_{c}, \mathbf{Q}_{\tau}\right\}$, and $\operatorname{diag}(\bullet)$ is diagonal matrix.

The scalar measurement model follows from the received signal of (1) by

$$
z(l)=h(\mathbf{x}(l))+n(l)
$$

where the measurement $y_{l}=y(l)=r(l)$, and

$$
h(\mathbf{x}(l))=\sum_{k=1}^{K} \sum_{i=1}^{M} c_{k, i}(l) d_{k, m(l)} a_{k}\left(l T_{s}-m(l) T_{b}-\tau_{k, i}(l)\right)
$$

The scalar measurement $z(l)$ is a nonlinear function of the state $\mathbf{x}(l)$. Hence, our goal is to find the $2 K \times 1$ joint estimator $E\left\{\mathbf{x}(l) \mid \mathbf{R}^{l}\right\}$ with estimated error covariance

$$
\mathbf{P}=E\left\{[\mathbf{x}(l)-\hat{\mathbf{x}}(l \mid l)]\left[[\mathbf{x}(l)-\hat{\mathbf{x}}(l \mid l)]^{T} \mid \mathbf{R}^{l}\right\}\right.
$$

\section{Particle Filtering}

\subsection{Implementing the PF}

A particle filter allows for a complete representation of the a posteriori distribution of the states, so that any statistical estimates, such as the mean, modes, kurtosis and variance, can be easily computed. They can therefore, deal with any nonlinearities or distributions. Particle filters rely on importance sampling and, as a result, require the design of proposal distributions that can approximate the a posteriori distribution reasonably well. The most common strategy is to sample from the probabilistic model of the states evolution. We are in a position to compute the particle filter algorithm [1]. The basic procedure to construct a particle filter is shown below. The detailed derivations are presented in [1] and [3]. The pseudo-code of a generic particle filter can now be presented.

1) Initialization: $t=0$

For $i=1, \ldots, N$, draw the states $\mathbf{x}_{0}^{(i)}$ from the prior $p\left(\mathbf{x}_{0}\right)$.

2) For $t=1,2, \ldots$

(1) Importance sampling step

- For $i=1, \ldots, N$, sample $\hat{\mathbf{x}}_{t}^{(i)} \sim q\left(\mathbf{x}_{t} \mid \mathbf{x}_{0: t-1}^{(i)}, y_{1: t}\right)$

- For $i=1, \ldots, N$, evaluate the importance weights up to a normalizing constant 


$$
\mathbf{w}_{t}^{(i)}=\mathbf{w}_{t-1}^{(i)} \frac{p\left(y_{t} \mid \hat{\mathbf{x}}_{t}^{(i)}\right) p\left(\hat{\mathbf{x}}_{t}^{(i)} \mid \hat{\mathbf{x}}_{t-1}^{(i)}\right)}{q\left(\hat{\mathbf{x}}_{t}^{(i)} \mid \mathbf{x}_{0: t-1}^{(i)}, y_{1: t}\right)}
$$

- For $i=1, \ldots, N$, normalize the importance weights:

$$
\tilde{\mathbf{w}}_{t}^{(i)}=\mathbf{w}_{t}^{(i)} / \sum_{j=1}^{N} \mathbf{w}_{t}^{(j)}
$$

(2) Resampling

- Multiply/Suppress samples $\hat{\mathbf{x}}_{0: t}^{(i)}$ with high/low importance weights $\widetilde{\mathbf{w}}_{t}^{(i)}$, respectively, to obtain $N$ random samples $\mathbf{x}_{0: t}^{(i)}$ approximately distributed according to $p\left(\mathbf{x}_{0: t}^{(i)} \mid y_{1: t}\right)$.

- For $i=1, \ldots, N$, set $\mathbf{w}_{t}^{(i)}=\widetilde{\mathbf{w}}_{t}^{(i)}=1 / N$

(3) Output: The output of the algorithm is a set of samples that can be used to approximate the a posteriori distribution as follows

$$
p\left(\mathbf{x}_{0: t} \mid y_{1: t}\right)=1 / N \sum_{i=1}^{N} \delta_{\mathbf{x}_{0: t}^{(i)}}\left(d \mathbf{x}_{0: t}\right)
$$

(4) One obtains straightforwardly the following estimate of $E\left[g_{t}\left(\mathbf{x}_{0: t}\right)\right]$

$$
E\left[g_{t}\left(\mathbf{x}_{0: t}\right)\right]=1 / N \sum_{i=1}^{N} g_{t}\left(\mathbf{x}_{0: t}^{(i)}\right)
$$

where the variables $\mathbf{w}_{t}$ is known as the normalized importance weights and $y_{t}$ is measurement signal and the a posteriori density $p\left(\mathbf{x}_{0: t} \mid y_{1: t}\right)$, where $\mathbf{x}_{0: t}=\left\{\mathbf{x}_{0}, \mathbf{x}_{1}, \cdots, \mathbf{x}_{t}\right\}$ and $y_{1: t}=\left\{y_{1}, y_{2}, \cdots, y_{t}\right\}$, constitutes the complete solution to the sequential estimation problem.

For example, letting $g_{t}\left(\mathbf{x}_{0: t}\right)=\mathbf{x}_{0: t}$ yields the optimal MMSE estimate $\hat{\mathbf{x}}_{0: t}=E\left[\mathbf{x}_{0: t} \mid y_{1: t}\right]$. The particles $\mathbf{x}_{0: t}^{(i)}$ are assumed to be independent and identically distributed (i.i.d.) for the approximation to hold. It is usually difficult to takes samples from given a posteriori distribution. Bayesian theory proves to result in another useful concept, the proposal distribution, $q\left(\mathbf{x}_{0: t} \mid y_{1: t}\right)$, for the Monte Carlo Estimation calculation[1]. Here, the particle filters considered are three resampling schemes. The PF algorithm can be easily derived from the SIS algorithm by an appropriate choice of : (i) The importance density: $q\left(\mathbf{x}_{t} \mid \mathbf{x}_{k-1}^{(i)}, y_{1: t}\right)$ is chosen to be the prior density $p\left(\mathbf{x}_{t} \mid \mathbf{x}_{t-1}^{(i)}\right)$, and (ii) Resampling step: to be applied at every time index. The above choice of importance density implies that we need samples from $p\left(\mathbf{x}_{k} \mid \mathbf{x}_{k-1}^{(i)}\right)$. Thus, the importance weights evaluate $\mathbf{w}_{t}^{(i)}=p\left(y_{t} \mid \mathbf{x}_{t}^{(i)}\right)$. As the importance sampling density for the PF is independent of measurement $y_{t}$, the state space is explored without any knowledge of the observations. As resampling is applied at each iteration, this can result in rapid loss of diversity in particles. However, the PF method does have the advantage that the importance weights are easily evaluated and the importance density can be easily sampled. 


\subsection{Resampling Schemes}

The particle filters rely on sequential importance sampling (SIS). The SIS algorithm discussed so far has a serious limitation: the variance of the importance weights increases stochastically over time. To avoid the degeneracy of the SIS simulation method, a resampling (selection) stage may be used to eliminate samples with low importance weights and multiply samples with high importance weights. It is possible to see an analogy to the steps in genetic algorithms [4]. A selection scheme associates to each particle $x_{0: t}^{(i)}$ a number of "children", say $N_{i} \in \mathrm{N}$, such that $\sum_{i=1}^{N} N_{i}=N$. Several selection schemes have been proposed in the literature. These schemes satisfy $E\left(N_{i}\right)=N \widetilde{w}_{t}^{(i)}$ but their performance varies in terms of the variance of the particles $\operatorname{var}\left(N_{i}\right)$. Results in [5] indicate that the restriction $E\left(N_{i}\right)=N \tilde{w}_{t}^{(i)}$ is unnecessary to obtain convergence results. So it is possible to design biased but computationally inexpensive selection schemes. We will now present a number of selection or resampling schemes, namely: Multinomial Resampling (MR), Residual Resampling (RR) and Minimum Variance Resampling (MVR). We found that the specific choice of resampling scheme affects the performance of the particle filter.

\subsubsection{Multinomial Resampling}

Resampling involves mapping the Dirac random measure $\left\{x_{0: t}^{(i)}, \widetilde{w}_{t}^{(i)}\right\}$ into an equally weighted random measure $\left\{x_{0: t}^{(j)}, N^{-1}\right\}$. This can be accomplished by sampling uniformly from the discrete set $\left\{x_{0: t}^{(i)} ; i=1, \cdots, N\right\}$ with probabilities $\left\{\tilde{w}_{0: t}^{(i)} ; i=1, \cdots, N\right\}$. Gordon [6] gave a mathematical proof of this result. After constructing the cumulative distribution of the discrete set, a uniformly drawn sampling index $i$ is projected onto the distribution range and then onto the distribution domain. The intersection with the domain constitutes the new sample index $j$. That is, the vector $x_{0: t}^{(j)}$ is accepted as the new sample. Clearly, the vectors with the larger sampling weights will end up with more copies after the resampling process.

Sampling $N$ times from the cumulative discrete distribution $\sum_{i=1}^{N} \tilde{w}_{t}^{(i)} \delta_{x_{0: t}^{(i)}}\left(d x_{0: t}\right)$ is equivalent to drawing $\left\{N_{i} ; i=1, \cdots, N\right)$ from a multinomial distribution with parameters $N$ and $\tilde{w}_{t}^{(i)}$. As we are sampling from a multinomial distribution, the variance is $\operatorname{var}\left(N_{i}\right)=N \tilde{w}_{t}^{(i)}\left(1-\tilde{w}_{t}^{(i)}\right)$. As pointed out in [7], it is possible to design selection schemes with lower variance.

\subsubsection{Residual Resampling}

This procedure involves the following steps [4]. Firstly, set $\tilde{N}_{i}=\left\lfloor N \tilde{w}_{t}^{(i)}\right\rfloor$. Secondly, perform an SIR procedure to select the remaining $\bar{N}_{t}=N-\sum_{i=1}^{N} \tilde{N}_{i}$ samples with new weights $w_{t}^{(i)}=\bar{N}_{t}^{-1}\left(\widetilde{w}_{t}^{(i)} N-\tilde{N}_{i}\right)$. Finally, add the results to the current $\tilde{N}_{i}$. For 
this scheme, the variance $\left(\operatorname{var}\left(N_{i}\right)=\bar{N}_{i} w_{t}^{\prime(i)}\left(1-w_{t}^{\prime(i)}\right)\right)$ is smaller than the one given by the SIR scheme. Moreover, this procedure is computationally cheaper.

\subsubsection{Minimum Variance Resampling}

This strategy includes the stratified/systematic sampling procedures introduced in [5]. One samples a set of $N$ points $U$ in the interval $[0,1]$, each of the points a distance $N^{-1}$ apart. The number of children $N_{i}$ is taken to be the number of points that lie between $\sum_{j=1}^{i-1} \tilde{w}_{t}^{(j)}$ and $\sum_{j=1}^{i} \tilde{w}_{t}^{(j)}$. This strategy introduces a variance on $N_{i}$ even smaller than the residual resampling scheme, $\left(\operatorname{var}\left(N_{i}\right)=\bar{N}_{i} w_{t}^{\prime(i)}\left(1-\bar{N}_{t} w_{t}^{\prime(i)}\right)\right)$

\section{Simulation}

We now examine the performance of the PF for making parameter estimates for a multiuser detector. We compare the PF-based estimator with among three resampling schemes. The multipath coefficients of the channel can be generated using Clarke and Gans Fading Model [8, Chap 4], which provides taps with the appropriate distributions and near the correct tap autocorrelations, although the taps are somewhat correlated. For simplification purposes, we consider no multipath. For the state model, the augmented state transition matrix of (5) was chosen to be $\mathbf{F}=0.999 \mathbf{I}$. Also the process noise covariance matrix was $\mathbf{Q}=0.001 \mathbf{I}$

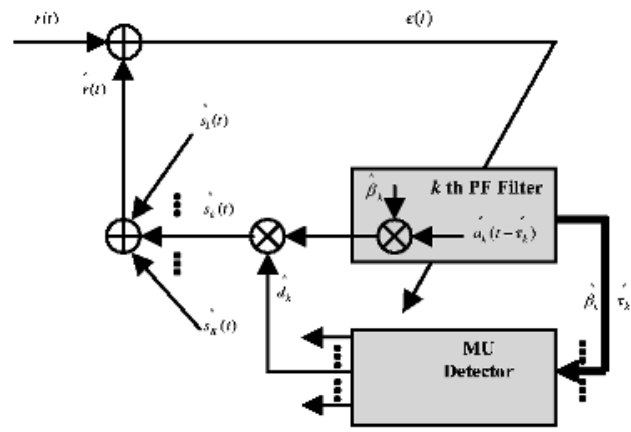

Fig. 1. Operation of multiuer parameter estimation receiver

We simulate a two-user scenario where the users' PN spreading codes are chosen from the set of Gold codes of length 31 and generated by the polynomials $x^{5}+x^{2}+1$ and $x^{5}+x^{4}+x^{3}+x^{2}+1$. The SNR (Signal-to-Noise Ratio) at the receiver of weaker user is $10 \mathrm{~dB}$. The Near-Far Ratio is $20 \mathrm{~dB}$. Oversampling factor (sample/chip) is 2. The SNR for the weaker user is set at $10 \mathrm{~dB}$. One aspect about using PF is that they require proper initialization. Depending on the problem, the initial guesses may need to be close to the correct value for convergence. For the simulation results, we 
assume such an initial estimator is used to start the tracking algorithm fairly close to the true values. Furthermore, we note that the data bits, $d_{k, m}$, are not included in the estimation process, but are assumed unknown a priori. In the simulations, we assume that the data bits are available from decision-directed adaptation, where the symbols $d_{k, m}$ are replaced by the decisions $\hat{d}_{k, m}$ shown in Fig. 1 .

The tracker for a two-user system is simulated for a fading channel where the channel coefficients are time varying, but the delays remain constant. A simple channel model is assumed for each user with a single tap (flat fading). Furthermore it is assumed that each user is moving with a Doppler frequency of $200 \mathrm{~Hz}$ for User 1 and $300 \mathrm{~Hz}$ for User 2. The fading for in-phase and quadrature components of the the channel coefficients for each user was implemented by IFFT and normalized so that the average power is unity. The sampling time is taken as $T_{C}=1 /(1.2288 \mathrm{Mbps} \times 2)$ and The bit rate is assumed to be $1 / T_{b}=9600 \mathrm{bps}$ with processing gain 31 .

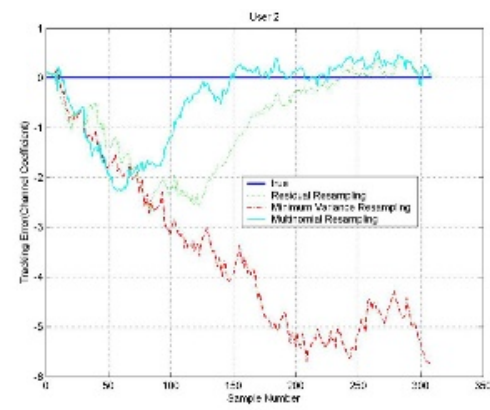

(a)

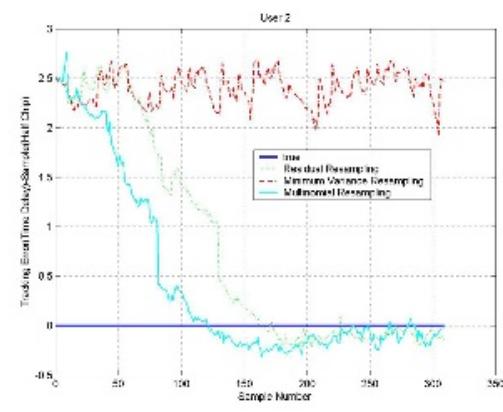

(b)

Fig. 2. Parameter estimation errors for (a) channel amplitudes and (b) time delays with a nearfar ratio of $0 \mathrm{~dB}$

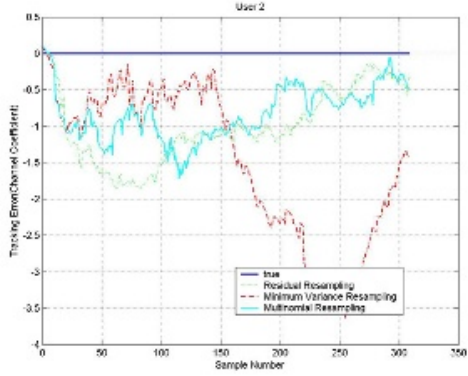

(a)

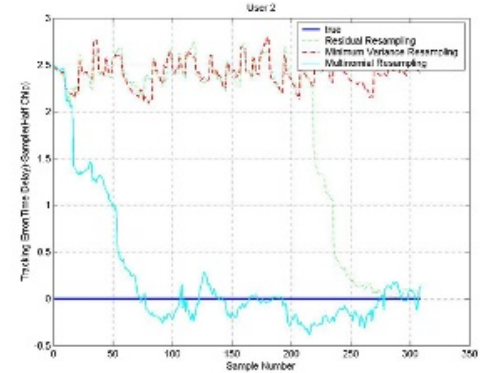

(b)

Fig. 3. Parameter estimation errors for (a) channel amplitude and (b) time delays with near-far ratio of $20 \mathrm{~dB}$ 
Fig. 2 shows the estimation error for channel coefficients and time delays with imperfect power controlled using the Multinomial Resampling, Residual Resampling and Minimum Variance Resampling, respectively. As the figure indicates, the estimator/tracker with $\mathrm{MR}$ and $\mathrm{RR}$ is able to accurately track the time-varying channel coefficients of each user, even for fast fading rates. But MVR is diverge. The results for the same set of assumptions, but with a near-far ratio of $20 \mathrm{~dB}$ are shown in Fig. 3.

Again, the estimator is able to accurately converge to the correct values of the parameters for both the MR and RR. But MR algorithm indicates to converge faster RR.

It is seen that a user is able to accurately converge to the correct delays and channel coefficient for PF with MR and RR scheme. PF with MR scheme indicates to converge faster and it has smaller mean squared error (MSE).

\section{Conclusions}

We have presented a parameter estimator based on the RPF that are capable of estimating channel coefficients and time delays in MAI (near-far ratio $=20 \mathrm{~dB}$ ). The PF with MR has been demonstrated to have superior performance over RR and MVR scheme. PF with MR can provide a better alternative to nonlinear filtering than RR or MVR schemes since it possible to design selection schemes with lower variance. Computer simulations also show that it provides a more effective technique for tracking time-varying amplitudes and delays in CDMA communication systems than RR and MVR. Furthermore the PF with MR estimator is shown to have the ability to converge to the user's true coefficients and time delays for a near-far ratio of $20 \mathrm{~dB}$.

\section{References}

1. Rudolph van der Merwe, Arnaud Doucet, Nando de Freitas, Eric Wan, "The Unscented Particle Filter," Oregon Graduate Institute Electrical and Computer Engineering, OR 97006, USA, August 16, 2000.

2. James J. Caffery, Wireless Location in CDMA Cellular Radio Systems, Kluwer Academic Publishers, 2000.

3. J.S. Kim and D.R. Shin, "Multiuser CDMA Parameters Estimation by Recursive Particle Filter", Multiuser CDMA Parameters Estimation by Recursive Particle Filtering(RPF), The 2003 Int'l Multiconference in Computer Science and Engineering, June 23 26, 2003, pages:444 447.

4. Higuchi, T. "Monte Carlo filter using the genetic algorithm operators," Journal of Statistical Computation and Simulation 59, 1997, pages: 1-23.

5. Kitagawa, G. "Monte Carlo filter and smoother for non-Gaussian nonlinear state space models," Journal of Computational and Graphical Statistics, 1996, pages: 1-25.

6. Gordon, N. J. Bayesian Methods for Tracking, PhD thesis, Imperial College, University of London, 1994.

7. Liu, J. S. and Chen, R. "Sequential Monte Carlo methods for dynamic systems," Journal of the American Statistical Association, 1998, pages:1032-1044.

8. Theodore S. Rappaport, Wireless Communications, IEEE Press, 1996. 